\title{
Influence of sport mouthguards on the ecological factors of the children oral cavity
}

Simonetta D'Ercole ${ }^{1,4^{*}}$, Diego Martinelli ${ }^{2}$ and Domenico Tripodi ${ }^{3}$

\begin{abstract}
Background: The use of fixed and/or removable dental devices is an attributable factor that may affect the oral cavity homeostasis. The aim of this study was to monitor the oral environmental changes caused by dental devices, as sports mouthguards with the aid of a chair-side test.

Methods: Sixty children with sports-mouthguards were analyzed at baseline (T0), after 6 months of dental devices use (T1), after a year (T2) and after almost 6 months without using it (T3). At T0, a clinical monitoring was performed and the DMFT index was recorded. At each time of observation, the following parameters were recorded: FMPS, FMBS, unstimulated-flow rate, saliva consistency, resting $\mathrm{pH}$, stimulated saliva, buffer capacity, the CFU/ml of Streptococcus mutans.

Results: In 60 subjects, mean age $9.9 \pm 1.2$, mean value of DMFT $1.55 \pm 1.29$, dmf-t $3.43 \pm 1.21$, FMPS and FMBS values increased significantly at $\mathrm{T} 2$. The values of unstimulated flow rate vary significantly within the observation times. The $\mathrm{pH}$ value and the buffering capacity reduced significantly at T2. The tests for the detection of S. mutans were negative in all the subjects in several observation times. All patients regularly used fluoridated toothpaste and comply with normal standards of oral hygiene; but over time the patients lost their initial motivation.

Conclusions: Sport treatment with dental devices dues to changes in oral ecological factors: increases FMPS, FMBS and reduces the buffering capacity and the salivary $\mathrm{pH}$. The use of removable devices increases the retentive plaque surfaces and inhibits the protective effect of saliva.

The so-called "chair-side" tests were able to easily monitor patients and to determine the risk of oral disease during sport treatment.
\end{abstract}

Keywords: Sport mouthguards, Dental devices, Salivary test, Streptococcus mutans

\section{Background}

The dental biofilm is a complex structure whose formation is influenced by many factors, which can alter both the structure and formation process. Normally the plaque coexists in the oral cavity in a sort of balance between activities of the microorganisms and host defenses. Pathogens can destroy this balance, which if disturbed can move towards the two main oral diseases: dental caries and periodontal disease. The use of fixed and/or removable dental devices is an attributable factor that may affect the balance.

\footnotetext{
* Correspondence: simonetta.dercole@virgilio.it

'Departments of Sperimental and Clinical Sciences, Dental School, University "G. D'Annunzio" of Chieti-Pescara, Chieti, Italy

${ }^{4}$ Oral Microbiology, Department of Sperimental and Clinical Sciences, University "G. d'Annunzio" Chieti- Pescara, Via dei Vestini, 3166100 Chieti, Italy

Full list of author information is available at the end of the article
}

Sport mouthguards were dental devices that have been utilized by athletes who recognized the need for oral protection during their sports activities and Ethylene Vinyl Acetate (EVA) has become widely accepted as a mouthguard material [1-4].

Recent studies of hockey and football players have shown that "boil and bite" protective athletic mouthguards harbor a range of pathogenic and opportunistic bacteria, yeasts, and molds $[5,6]$.

Ultrastructural analyses by SEM reveal that the microorganisms can be found both on the surfaces and in the porosities of the polymerized boil and bite mouthguard material [7]. A recent study over the course of a season confirmed that football mouthguards increased the number and severity of oral mucosal injuries [5,7]. Previous 
studies found that some commercially available mouthguards were contaminated before use [5].

No data are availables about the association between custom-made sport mouthguards and the changes occurring in the oral ecosystem; on the contrary several studies have been performed on patients wearing removable and fixed orthodontic appliances [8-10]. The relationship between the use of orthodontic appliances and the development of caries, however, is still under discussion. Some authors reported that the orthodontic treatment alone can not increase the incidence of caries $[11,12]$ while others demonstrated an increase of enamel lesions or proximal caries in correlation with the use of fixed or removable devices [13-15]. Certainly, the orthodontic treatment involves the changes in oral microflora, increasing the cariogenic microorganisms in plaque and saliva. The effect of fixed orthodontic appliances on the colonization of cariogenic microorganisms has been well established; on the contrary it is not clear whether the removable appliances can increase the levels of pathogenic Streptococci in the oral cavity $[16,17]$. Few studies reported about the association between orthodontic treatment and the degree of salivary flow, and the buffering capacity of saliva, and still cover all fixed orthodontic treatment $[8-10,18,19]$. There is now ability to easily monitor patients and to determine the risk of caries and periodontal disease, before and during sport treatment, thanks to the introduction of the so-called "chair-side"tests. They allow identifying changes that occur in the oral cavity during treatment and are mainly based on research of Streptococcus mutans and Lactobacillus spp and determination of salivary characteristics $(\mathrm{pH}$, buffer capacity, etc.) [20-24].

The purpose of this study was to monitor environmental changes of the oral cavity by the determination of clinical, salivary and bacterial markers, before, during and after sport treatment with mouthguards, with the aid of chair-side test.

\section{Methods}

\section{Study population}

60 children were selected, 27 males, 33 females, aged between 8 and 12 years, referred to the Department of Medical, Oral and Biotechnological Sciences, University of Chieti, in the period 2010-11.

The following inclusion criteria were considered:

1. Age: $7-14$ years $(7<$ age $<14)$

2. Absence of any active carious lesion

3. Dental Care not in progress

4. Need for sport treatment with mouthguards.

Patients were excluded from the study if they met any of the following exclusion criteria:
1. Periodontitis

2. Partial or total removable prosthesis

3. Poor medical conditions (diabetes, asthma), systemic antibiotics, or local antimicrobials during 3 months preceding the sport treatment placement, patients under current medication affecting the saliva flow rate.

During the study, a patient who started a medication affecting the saliva flow rate or the biofilm composition was immediately excluded.

The selected subjects participated voluntarily in the study. Patients and their parents were first given oral and written information on the study's purpose. Informed consent was given by signing a protocol. (Privacy Law DL 196/2003). In this study, the approval from the Ethical Committee it's not reported because that's not required for works that are based on research protocol on medical devices already used in the clinical protocols approved from the Department for the medical use.

A self-administered questionnaire was used to obtain data concerning a pathological complete history, a history of hard and soft tissues of the oral cavity, a family history, oral hygiene practices, fluoride intake and snacking habits (use of supplements, dietary information, such as intake of drinks, fruit juices and consumption of chocolates).

\section{Clinical monitoring}

Patients were analyzed at baseline, before starting sport therapy with custom-made EVA mouthguards (T0), after 6 months of therapy (T1), after a year (T2) and finally after almost 6 months without using the device (T3).

At T0, a clinical monitoring was performed and on each patient were recorded the number of decayed $(D)$, missing (M) and filled (F) teeth (T) (DMFT) and dmft to assess caries prevalence according to WHO criteria [25]. In addition, an oral examination of intraoral mucosal was performed and assessed the presence/absence of bad habits and/or parafunctional habits. During the first visit were explained to each patient the rules for a proper use of toothbrush and toothpaste. Each patient has repeated the indications received so the clinician was able to correct any inaccuracy.

At each time of observation, the following parameters were recorded: FMPS (Full Mouth Plaque Score) and FMBS (Full Mouth Bleeding Score) to evaluate the oral hygiene and periodontal status. The patients avoided eating or drinking and they didn't effect the tooth brushing at least 2 hours before taking the samples in all stages. All procedures were done by one calibrated researcher, and commercial kits were used according to the manufacturer's instructions. 


\section{Saliva analysis}

Then on each patient, in the 4 time points, the following indices were determined with the aid of the GC Saliva Check Kit (GC Corp., Belgium). The first was performed by a visual inspection of level of hydration. The unstimulated flow rate was measured visually, noting the time taken for a salivary droplet to form on the lower lip. A time greater than $60 \mathrm{~s}$ was considered as abnormally low (according to the manufacturer's instructions). Then visually was analyzed the saliva consistency in the oral cavity. A sticky frothy saliva residue was considered as increased viscosity.

Subjects were then asked to pool their saliva in the floor of the mouth and then expectorate over $30 \mathrm{~s}$ into the collection cup. A pH tests trip was dipped in the saliva and the colour used to estimate the $\mathrm{pH}$ comparing with the testing chart available in the kit. To stimulate saliva it was given to the subjects a paraffin wax to chew and saliva was collected for $5 \mathrm{~min}$ in a measuring cup and the volume was calculated. From the collected saliva a drop was placed on a buffering strip, left for $5 \mathrm{~min}$ and then measured on the $\mathrm{pH}$ scale provided.

\section{Bacterial markers}

To estimate the number of colony-forming units of Streptococcus mutans counts (SM) per milliliter of saliva (CFU/mL) GC Saliva-Check Mutans (GC Corp., Belgium) was used.

This test kit provides a semi-quantitative evaluation of the level of S.mutans in the saliva in 15 minutes by using monoclonal antibodies.

\section{Statistical analysis}

For statistical analysis the Matched-Pairs Signed-Wilkoxonrank test have been used. Data are shown as Mean values (Standard Deviation-SD), and for all analyses a P-value of less than 0.05 was considered significant.

\section{Results}

As shown in Table 1, sixty subjects, mean age 9.9(1.2), with mean value of DMFT 1.55 (1.29) and dmf-t 3.43 (1.21) participated to this study. Allergies, in particular to nickel and resins-define, which could affect the individual response, were almost absent. In addition, information obtained indicated that none of the 60 children was suffering from food allergies or medication and only one of them had undergone surgery. All patients were not receiving regular fluoride but they regularly used fluoridated toothpaste. From the data obtained about the eating habits, it was shown that the majority of subjects took regular carbohydrates in the diet (pasta, bread, cakes, candies, etc.), but it doesn't abuse of it (data not shown).
Table 1 Demographic characteristics of the studied population

\begin{tabular}{cc}
\hline & T0 \\
\hline Average age & $9.9(1.2)$ \\
Sex & $27 \mathrm{M} 33 \mathrm{~F}$ \\
DMFT & $1.55(1.29)$ \\
dmft & $3.43(1.21)$ \\
Allergies & Yes 20\% \\
Sealing & Yes $40 \%$ \\
Intake of fluoride & No \\
Use of fluoridated toothpaste & Yes 100\%
\end{tabular}

T0 = baseline.

With regard to clinical indices (Figure 1), the FMPS value increased significantly from $\mathrm{T} 0$ to $\mathrm{T} 2$ and then restabilized at T3, about to the levels of $\mathrm{T} 1$. The value of FMBS unchanged between $\mathrm{T} 0$ and $\mathrm{T} 1$ and reached values statistically significant at T2 and newly improved at T3.

The results of saliva and bacteria analysis are shown in Figure 2 and Table 2. Within the four observation times there was a significant variation of the values of unstimulated flow rate, who suffered a statistically significant increase to $\mathrm{T} 1$, and a statistically significant reduction at T2 and T3 compared to T1, so as to return to baseline values. The saliva consistency does not undergo significant changes over time. The $\mathrm{pH}$ value of the baseline, however, undergoes a significant decrease in $\mathrm{T} 2$ to return to the previous levels in T3. The amount of saliva produced under stimulation in 5 minutes didn't vary significantly at $\mathrm{T} 0, \mathrm{~T} 1$ and $\mathrm{T} 2$, but the difference between $\mathrm{T} 1$ and $\mathrm{T} 3$ was statistically significant. The buffering capacity was drastically reduced in a statistically significant way at $\mathrm{T} 2$.

The tests for the detection of Streptococcus mutans were negative in all the subjects on several observation times (Table 2).

The Table 3 shows that patients comply with normal standards of oral hygiene, both in regard to brushing the teeth that cleaning removable appliance that actually improved during the first months of therapy, but over time

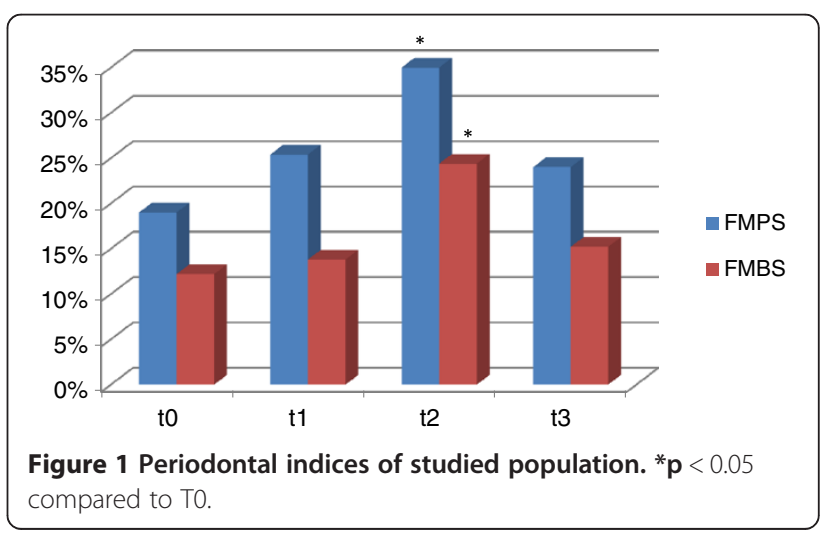




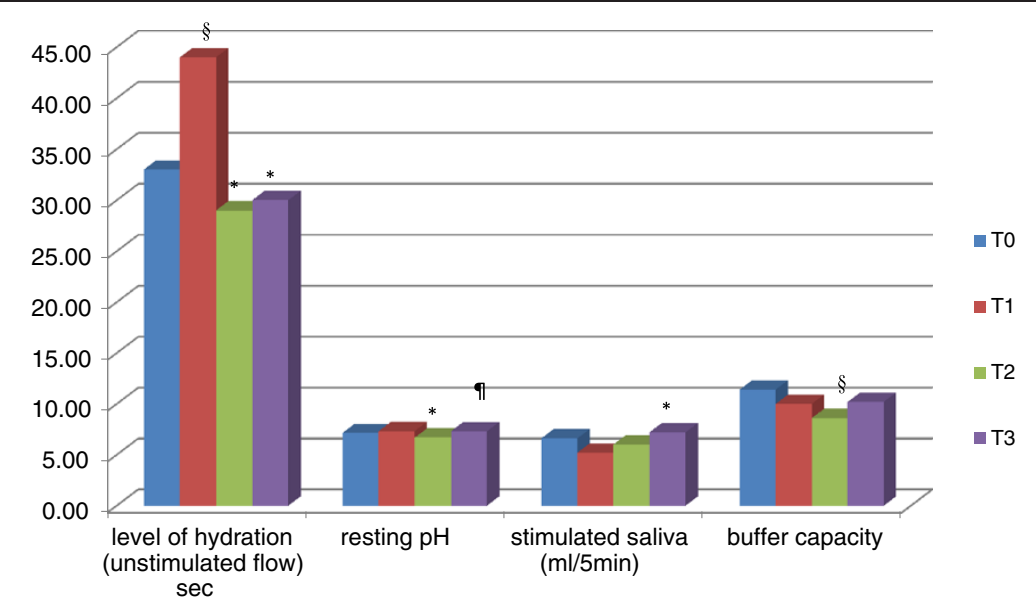

Figure 2 Salivary and microbiological factors examined with the aid of a chair-side salivary test. T0 = baseline. T1 = patients carrying the sport mouthguards from 6 months. $\mathbf{T} 2$ = patients carrying the sport mouthguards from 1 year. $\mathbf{T} 3=$ patients who completed sport therapy for 6 months. ${ }^{\S} \mathbf{p}<0.05$ compared to T0. ${ }^{*} \mathbf{p}<0.05$ compared to T1. ${ }^{\text {p }} \mathbf{p}<0.05$ compared to T2.

the patients lost their initial motivation (see difference between $\mathrm{T} 1$ and $\mathrm{T} 2$ ).

\section{Discussion}

This longitudinal study shows the clinical, salivary and bacterial changes that occurred in the oral cavity during and after treatment with custom-made sport mouthguards. Normally, the oral cavity has the ability to adapt it to the presence of a foreign body, increasing the salivary flow which contributes to autoclysis and altering the composition of saliva to maintain high values of $\mathrm{pH}$ and buffering capacity, so as to prevent the colonization of microorganisms potentially pathogenic by the refusal of the optimal environmental conditions.

The advent of a simple chair-side saliva check kit allows to easily monitor patients and to determine the risk of caries and periodontal disease before and during sport treatment.

The unstimulated salivary flow, as observed in this study, increased initially after 6 months of therapy in response to a foreign body, but after 1 year of therapy decreased significantly. The amount of saliva produced under stimulation in 5 minutes (salivary flow) didn't vary significantly between the baseline and during all the treatment. In contrast, 6 months after the end of treatment there was a statistically significant increase compared with
6 months of therapy, confirming the results obtained from Sanpei and colleagues [19] who showed how changes in salivary flow that occur in the period of orthodontic treatment were statistically insignificant. On the contrary Chang and colleagues [8], Ulukapi and colleagues [18], Cheng and colleagues [9] showed that the salivary flow increased significantly after the orthodontic devices positioning.

The results of this study demonstrated that the use of removable appliances, as custom-made mouthguards, lead to a significant lowering of the $\mathrm{pH}$ scale defined in moderately acid after a year of therapy, necessitating later than 6 months after therapy to return to normal levels. The $\mathrm{pH}$ decreased as the mouthguard increased the areas and retentive surfaces against the plaque, thus causing high acid levels due to high concentration of hydrogen ions in the oral cavity.

The buffering capacity of saliva prevents the colonization of pathogenic microorganisms in the mouth and neutralizes acids produced by acidogenic bacteria, thus preventing the demineralization of enamel. This can be regarded as one of the best indicators of risk, as revealed bythe answer of the host [10]. Patients with high buffering capacity are often resistant to the development of caries, because the high response of the host can be compensatory against the activity of the lesion.

Table 2 Salivary consistence and presence/absence of S. mutans in the population studied

\begin{tabular}{ccccc}
\hline & T0 & T1 & T2 & T3 \\
\hline Saliva consistency & Normal & Normal & Increased in 20\% of cases & Increased in 20\% of cases \\
Streptococcus mutans & Negative & Negative & Negative & Negative \\
\hline
\end{tabular}

T0 = baseline.

$\mathbf{T 1}=$ patients carrying the sport mouthguards from 6 months.

$\mathbf{T} 2$ = patients carrying the sport mouthguards from 1 year.

T3 $=$ patients who completed sport therapy for 6 months. 
Table 3 Oral hygiene habits of the studied population

\begin{tabular}{lcccc}
\hline & T0 & T1 & T2 & T3 \\
\hline Brushing/die & 2 times. & 2.2 times. & 1.6 times. & 1.8 times. \\
Cleaning device/die & ND & 2.2 times. & 1.6 times. & ND \\
\hline T0 $=$ baseline. & & & \\
$\mathbf{T 1}=$ patients carrying the sport mouthguards from 6 months. \\
T2 $=$ patients carrying the sport mouthguards from1 year. \\
T3 = patients who completed sport therapy for 6 months. \\
ND: Not Detected.
\end{tabular}

Studies on the salivary markers and their association with the prevalence of caries in adult populations have been widely performed in the past, but with mixed results $[8,9,18,19,26]$.

However, there seems to be a correlation between the saliva $\mathrm{pH}$ and the prevalence of early carious lesions, as well as between the buffering capacity and potential activity in lesions of moderate type. Varma and colleagues [26] reported that the $\mathrm{pH}$ of the saliva can be considered a predictive factor in the development of pre-carious lesions. In the presence of active lesions, however, the buffer capacity can be of help in predicting the potential activity in the dentinal lesions present.

In this study, the buffering capacity of saliva was greatly reduced in a statistically significant way after a year of sport treatment with mouthguards.

The results showed that the dental devices does not influence the salivary flow, which has among other things, the function of providing the bicarbonate ions and thus to increase the $\mathrm{pH}$. On the contrary, in this case, in parallel, also the $\mathrm{pH}$ decreases as well as the buffering capacity, showing an altered response of the host in maintaining oral health in adverse situations and thus decreasing the protective and preventive activity of the saliva.

About the effect played by removable appliances, Batoni and colleagues [27] showed that the presence in the oral cavity of orthodontic removable devices leads to the creation of new retentive areas and surfaces adhesionand growth-promoting of S. mutans.

Glass and colleagues reported that the boil and bite mouthguards were contaminated by microorganisms and have the propensity to become a microbial reservoir. Therefore, they have the potential to produce oral and systemic diseases because the jagged, sharp areas of the posterior regions of typical mouthguards are in close proximity to the pterygoid plexus of veins and, so, the entire circulatory system [5-7].

In this study the use of the test "Saliva-Check Mutans GC" showed that levels of $S$. mutans remain unchanged during the study, in agreement with Sanpei and colleagues [19] and Lara-Carrillo and colleagues [10]. The test used in this study remained negative for all time, but the test in question presented low sensitivity and low quantitative discrimination, because based only on the presence/absence of the microorganism with level of cutoff equal to $5 \times 10^{5} \mathrm{CFU} / \mathrm{ml}$.

The microbiological test used in this study was easy to use and relatively inexpensive and thus it allows realizing a quick screening of populations at risk due to its own cut-off of reference, even if its accuracy was lower than that of the conventional cultivation method [28-30].

The use of sport mouthguards lead to a worsening of the oral health of analyzed patients, as demonstrated by a significant increase after one year of therapy of the clinical indices FMPS and FMBS, which would significantly improve with the suspension for 6 months of the sport treatment. The increase of the bacterial plaque was due both to changes in the characteristics of the saliva, which we have seen was able to facilitate the formation of the biofilm, both to the particular features of the device. The plaque has a tendency to accumulate in the retentive areas of springs, claps and polymeric-material baseplate $[7,31]$ that are sites where it can easily be the penetration of microorganisms due to the properties of the material itself. The EVA is a copolymer and when the material is prepared it's possible to observe the reactions monomerpolymer. If the process is not performed correctly, pores can appear on the surface facilitating the adhesion of microorganisms [1-3,32].

Based on these results, several preventive strategies for the changes that occur in the oral cavity of athletes should be implemented. The study of the quality, $\mathrm{pH}$ and buffering capacity of the saliva, the monitoring of the bacterial count, plaque and gingival inflammation can be an important part of a complete clinical control. Maintain a good oral hygiene during sport treatment is very difficult. Anyway in light of the possible consequences (development of tooth decay, gingival and periodontal disease) is crucial to give the patient proper oral hygiene instructions starting therapy and to monitor the course of therapy. Then, the tests must be repeated at regular intervals during treatment and the patient must be motivated with regular reminders of oral hygiene and controls in order to avoid the onset of disease, since, as demonstrated by this study, the motivation is lost in time and it's reduced the number of washes of teeth and device. Other preventive approaches require that the patient is informed about the potential of cariogenic foods and beverages and use of appropriate antimicrobial therapy and administration of fluoride. According to Benson and colleagues [33] the subministration of fluoride $0.05 \%$ through mouthwashes and topical application in the gel can help to prevent the development of the biofilm, but as demonstrated in this study in which patients were regularly using fluoride toothpaste, there is no influence on the ecological characteristics of the oral cavity. It should be recommended in any case the use of chlorhexidine, due to its widely demonstrated bactericidal 
activity. Disinfection with antimicrobial agents should obviously include not the onlyoral cavity but also the device [34,35]. Alternatively, as suggested by Arnold and colleagues [36] chlorhexidine can be incorporated in the matrix EVA that slowly released the antimicrobial agent into the oral cavity.

\section{Conclusions}

Sport treatment with custom-made mouthguards due to changes in ecological factors of the oral cavity, because increases FMPS and FMBS and reduces the buffering capacity and salivary $\mathrm{pH}$, thus inhibiting the protective effect of saliva. Furthermore, after a year of therapy there is a worsening of the clinical indices of the patient, who loses the motivation to maintain good oral hygiene. It's necessary to identify those at risk from the outset of therapy, and then still to monitor patients during all therapy.

The advent of simple chair-side check kits that allow to analyze the bacterial count, the labial hydration level of unstimulated saliva, quality, resting $\mathrm{pH}$ and buffering capacity of stimulated saliva, to be associated with clinical indices, necessary for the determination of plaque and gingival inflammation, it offers the possibility to follow very well during the time patients with sport mouthguards without incurring in future diseases.

\section{Abbreviations}

EVA: Ethylene Vinyl Acetate; DMFT: Number of decayed (D), missing (M) and filled (F) teeth (T); FMPS: Full mouth plaque score; FMBS: Full mouth bleeding score; CFU/mL: Number of colony-forming units per milliliter; SD: Standard deviation.

\section{Competing interests}

The authors declare that they have no competing interests.

\section{Authors' contributions}

SD has written the clinical protocol and the manuscript. DM has done the salivary test on the patients. DT has organized the research. All authors read and approved the final manuscript.

\section{Authors' information}

$\mathrm{SD}, \mathrm{DDS}, \mathrm{PhD}$, is a research fellow. DM, DDS, is a postgraduate student. DT, $\mathrm{MD}, \mathrm{DDS}$ is a professor.

\section{Funding}

This study was supported by the Department of Medical, Oral and Biotechnological Sciences, University "G. d'Annunzio" Chieti.

\footnotetext{
Author details

${ }^{1}$ Departments of Sperimental and Clinical Sciences, Dental School, University "G. D'Annunzio" of Chieti-Pescara, Chieti, Italy. ${ }^{2}$ Departments of Medical, Oral, and Biotechnological Sciences, Dental School, University "G. D'Annunzio" of Chieti-Pescara, Chieti, Italy. ${ }^{3}$ Unit of Pediatric Dentistry, Departments of Medical, Oral, and Biotechnological Sciences, Dental School, University "G. D'Annunzio" of Chieti-Pescara, Chieti, Italy. ${ }^{4}$ Oral Microbiology, Department of Sperimental and Clinical Sciences, University "G. d'Annunzio" Chieti- Pescara, Via dei Vestini, 3166100 Chieti, Italy.
}

Received: 7 April 2014 Accepted: 25 July 2014

Published: 5 August 2014

\section{References}

1. Knapik JJ, Marshall SW, Lee RB, Darakjy SS, Jones SB, Mitchener TA, Dela Cruz GG, Jones BH: Mouthguards in sport activities: history, physical properties and injury prevention effectiveness. Sports Med 2007, 37(2):117-144.

2. American Dental Association Division of Science. For the dental patient: Keep sports safe-wear a mouthguard. JADA 2012, 143(3):312.

3. Ogawa T, Yamasaki S, Honda M, Terao Y, Kawabata S, Maeda Y: Long-term survival of salivary streptococci on dental devices made of ethylene vinyl acetate. Int J Oral Sci 2012, 4(1):14-18.

4. Needleman I, Ashley P, Petrie A, Fortune F, Turner W, Jones J, Niggli J, Engebretsen L, Budgett R, Donos N, Clough T, Porter S: Oral health and impact on performance of athletes participating in the London 2012 Olympic Games: a cross-sectional study. Br J Sports Med 2013, 47(16):1054-1058.

5. Glass R, Bullard J, Goodson L, Conrad R: Microbial contamination of protective mouth-guards in hockey players: an in vivo study. Compend Cont Educ Dent 2001, 22:1093-1108.

6. Glass R, Wood C, Bullard J, Conrad S: Possible disease transmission by contaminated mouth-guards in two young football players. Gen Dent 2007, 55:436-440.

7. Glass RT, Conrad RS, Köhler GA, Warren AJ, Bullard JW: Microbiota found in protective athletic mouthguards. Sports Health 2011, 3(3):244-248.

8. Chang HS, Walsh $L$, Freer TJ: The effect of orthodontic treatment on salivary flow, pH, buffer capacity, and levels of mutans streptococci and lactobacilli. Aust Orthod J 1999, 15:229-234.

9. Cheng LL, Moor SL, Kravchuk O, Meyers IA, Ho CT: Bacteria and salivary profile of adolescents with and without cleft lip and/or palate undergoing orthodontic treatment. Aust Dent J 2007, 52:315-321.

10. Lara-Carrillo E, Montiel-Bastida NM, Sanchez-Perez L, Alais-Tavira J: Effect of orthodontic treatment on saliva, plaque and the levels of Streptococcus mutans and Lactobacillus. Med Oral Patol Oral Cir Bucal 2010, 15(6):924-929.

11. Wisth PJ, Nord A: Caries experience in orthodontically treated individuals. Angle Orthod 1977, 47:59-64.

12. Southard TE, Cohen ME, Ralls SA, Rouse LA: Effects of fixed-appliance orthodontic treatment on DMF indices. Am J Orthod Dentofacial Orthop 1986, 90:122-126.

13. Hollender $L$, Ronnerman A: Proximal caries progression in connection with orthodontic treatment. Swed Dent J 1978, 2:153-160.

14. Bjerlin K, Garskog B, Ronnerman A: Proximal caries increment in connection with orthodontic treatment with removable appliances. Br J Orthod 1983, 10:21-24

15. Ogaard B: Prevalence of white spot lesions in 19-year-olds: a study on untreated and orthodontically treated persons 5 years after treatment. Am J Orthod Dentofacial Orthop 1989, 96:423-427.

16. Petti S, Barbato E, Simonetti D'Arca A: Effect of orthodontic therapy with fixed and removable appliances on oral microbiota: a six-month longitudinal study. New Microbiol 1997, 20(1):55-62.

17. Jordan C, Leblanc DJ: Influences of orthodontic appliances on oral populations of mutans streptococci. Oral Microbiol Immunol 2002, 17:65-71.

18. Ulukapi H, Koray F, Efes B: Monitoring the caries risk of orthodontic patients. Quintessence Int 1997, 28(1):27-29.

19. Sanpei S, Endo T, Shimooka S: Caries risk factors in children under treatment with sectional brackets. Angle Orthod 2010, 80:509-514.

20. Lenčova E, Broukal Z, Spižek J: Salivary microbial tests for detection of cariogenic species. Folia Microbiol 2010, 55(6):559-568.

21. Chaussain C, Opsahl Vital S, Viallon V, Vermelin L, Haignere C, Sixou M, Lasfargues JJ: Interest in a new test for caries risk in adolescents undergoing orthodontic treatment. Clin Oral Invest 2010, 14:177-185.

22. Maldupa I, Brinkmane A, Mihailova A: Comparative analysis of CRT Buffer, GC saliva check buffer tests and laboratory titration to evaluate saliva buffering capacity. Stomatologija 2011, 13(2):55-61.

23. Robert JG: Salivary diagnostic tests. JADA 2012, 143(10 suppl):3S-5S

24. D'Amario M, Barone A, Marzo G, Giannoni M: Caries-risk assessment: the role of salivary tests. Minerva Stomatol 2006, 55(7-8):449-463.

25. Oral Health Survey Basic Methods. World Health Organization; 1997.

26. Varma S, Banerjee A, Bartlett D: An in vivo investigation of associations between saliva properties, caries prevalence and potential lesion activity in an adult UK population. J Dent 2008, 36:294-299. 
27. Batoni G, Pardini M, Giannotti A, Ota F, Giuca MR, Gabriele M, Campa M, Senesi S: Effect of removable orthodontic appliances on oral colonisation by mutans streptococci in children. Eur J Oral Sci 2001, 109(6):388-392.

28. D'Ercole S, Ristoldo F, Quaranta F, Amaddeo P, Tripodi D: Analysis of oral health status and of salivary factors in young soccer players: a pilot study. Medicina Dello Sport 2013, 66(1):71-80.

29. D'Ercole S, Catamo G, Piccolomini R: Diagnosis in periodontology: a further aid through microbiological tests. Crit Rev Microbiol 2008, 34(1):3-41.

30. Rego RO, Oliveira CA, Dos Santos-Pinto A, Jordan SF, Zambon JJ, Cirelli JA Haraszthy VI: Clinical and microbiological studies of children and adolescents receiving orthodontic treatment. Am J Dent 2010, 23(6):317-323.

31. Pietrzak P, Smiech-Slomkowska G: Evaluation of oral hygiene in patients treated with removable appliances. Czas Stomatol 2009, 62(9):728-734.

32. Lessa FCR, Ito IY, Faria G, Matsumoto MAN, Nelson-Filho P: In vivo evaluation of the bacterial contamination and disinfection of acrylic baseplates of removable orthodontic appliances. Am J Orthod Dentofacial Orthop 2007, 131(6):705-705.

33. Benson PE, Parkin N, Millet DT, Vine S, Shah A: Fluorides for the prevention of white spots on the teeth during fixed brace treatment. Cochrane Database Syst Rev 2004, 3, CD003809.

34. Peixoto IT, Enoki C, Ito IY, Matsumoto MA, Nelson-Filho P: Evaluation of home disinfection protocols for acrylic baseplates of removable orthodontic appliances: A randomized clinical investigation. Am J Orthod Dentofacial Orthop 2011, 140(1):51-57.

35. D' Ercole S, Tripodi D: The effect of swimming on oral ecological factors. J Biol Regul Homeost Agents 2013, 27(2):551-558.

36. Arnold RR, Wei HH, Simmons E, Tallury P, Barrow DA, Kalachandra S: Antimicrobial activity and local release characteristics of chlorhexidine diacetate loaded within the dental copolymer matrix, ethylene vinyl acetate. J Biomed Mater Res B Appl Biomater 2008, 86(2):506-513.

doi:10.1186/1472-6831-14-97

Cite this article as: $D^{\prime}$ Ercole et al: Influence of sport mouthguards on the ecological factors of the children oral cavity. BMC Oral Health 2014 14:97

\section{Submit your next manuscript to BioMed Central and take full advantage of:}

- Convenient online submission

- Thorough peer review

- No space constraints or color figure charges

- Immediate publication on acceptance

- Inclusion in PubMed, CAS, Scopus and Google Scholar

- Research which is freely available for redistribution 